\title{
Host Resistance to Parasitism: Do Older Tadpoles of Polypedates crucigers bear Higher Resistance towards Pleurolophocercous cercariae?
}

\author{
Pathirana N.U.K. ${ }^{1,2}$, Meegaskubura M. ${ }^{3}$, Rajakaruna R.S. ${ }^{1}$ \\ ${ }^{1}$ Department of Zoology, University of Peradeniya, Sri Lanka \\ ${ }^{2}$ Postgraduate Institute of Science, University of Peradeniya, Sri Lanka \\ ${ }^{3}$ Department of Molecular Biology \& Bio-technology, University of Peradeniya, Sri Lanka \\ *nuwpathirana@gmail.com
}

\begin{abstract}
Hosts increase their fitness by increasing resistance when exposed to parasites. In the context of tadpoles, the resistance can be conceived as the ability to protect themselves against parasitism by reducing parasite burden. Here we examined the age dependent resistance of the tadpoles of common hourglass tree frog (Polypedates cruciger), exposed to pleurolopho cercouscercariae (Trematoda: Digenea) under laboratory conditions. Eggs masses of $P$. cruciger were brought to the laboratory and the tadpoles were raised in glass tanks. Pleurolopho cercouscercariae released from freshwater snail, Melanoides tuberculata were exposed to tadpoles at 10, 20 and 30 days post-hatch (Gosner stages 27, 28/29, and 30/31, respectively) in a dose-dependent manner (10 cercariae per tadpole). After $1 \mathrm{~h}$ the number of cercariae that could not penetrate the tadpole was counted. The number of cysts, their locations in the host body and the survival of the tadpoles were recorded for five consecutive days post-exposure. Older tadpoles had a higher resistance to parasitism than the younger tadpoles. Overall, a significantly fewer number of cercariae penetrated the older tadpoles compared to the younger ones (10 days old tadpoles $=717 \pm 2.06 ; 20$ days $=666 \pm 2.42 ; 30$ days $=494 \pm 3.01$, One way ANOVA, $\mathrm{F}=24.46 ; p<0.001)$. Moreover, infection at 10 days posthatch stage resulted in significantly low survival $\left(60.0 \%\right.$; Chi square test, $\chi^{2}=38.960 ; p<$ 0.001 ) compared to the control, but not in 20 and 30 days post-hatch tadpoles (20 days: $73.3 \%, 30$ days; $93.3 \%$; chi square test $p>0.05$ ). An increase in the average number of cysts was observed for all treatments until the second day, after which the numbers decreased. Although, disappearing of cysts over time was higher in older tadpoles than in younger ones, it was not statistically significant compared with the other groups (One way ANOVA, $F=1.61$; $p>0.05)$. The older tadpoles have the ability to avoid penetration of cercariae and resist the already encysted parasites inside, showing an age dependent resistance by the tadpoles of $P$. cruciger to pleurolophocercous cercariae.
\end{abstract}

Keywords: Pleurolophocercous cercariae, Polypedates cruciger, Survival, Cysts, Resistance 